\title{
On the robustness of the computed torque technique in manipulator control $\dagger$
}

\author{
OLAV EGELAND $\ddagger$
}

Keywords: Robots, robustness analysis

In this paper, the robustness of the computed torque technique for manipulator control is investigated in the presence of model errors. The robustness analysis is performed in the frequency domain by means of the block Gerschgorin theorem. This theorem gives inclusion regions for the eigenvalues of the linearized state space model. The stability of the system close to a trajectory can then be investigated. The results indicate that relatively large errors in the non-linear feedback compensation of system non-linearities may be tolerated without affecting system stability. However, even small errors in the computed inertia matrix may result in instability.

\section{Introduction}

The state space equations of motion of an $n$-link robotic manipulator are nonlinear and contain a large number of terms. However, due to the special structure of the state space model, the non-linearities may be compensated for by non-linear feedback. In this way a linear time-invariant state space model consisting of $n$ decoupled double integrators is obtained. A controller for each of these $n$ double integrators may then be designed using linear control theory.

This is the well-known inverse dynamics or computed torque technique (Bejczy, 1974) when the problem is formulated in the joint space. The same technique has been applied in the task space by Luh, Walker and Paul (1980a) in their resolved acceleration control scheme and by Tarn, Bejczy, Isidori and Chen (1984). These control schemes have also been derived by Luo and Saridis (1985) by means of optimal control theory.

The computed torque technique is based upon the assumption that the nonlinear feedback compensation is exact and that the inertia matrix is known. The assumption will not hold in the presence of modeling errors or model simplifications. The question of the robustness of the resulting control system is therefore raised. This problem has been treated by Spong and Vidyasagar (1985), who employ the stable factorization technique to derive a controller that is stable in the presence of model uncertainty provided that the difference between the actual and computed inertia matrix is less than a given bound. The closed loop performance of the system is given by an explicit bound on the tracking error as a function of model uncertainty.

Received 23 March 1987.

† Copyright (C) 1986 I.E.E.E. Reprinted, with permission, from 1986 I.E.E.E. International Conference on Robotics and Automation, April 7-10, 1986, San Francisco, CA, pp. 12031208.

$\ddagger$ The Norwegian Institute of Technology, Division of Engineering Cybernetics, N-7034 Trondheim, Norway. 
In this paper, the robustness of the computed torque technique is analyzed in the frequency domain. The block Gerschgorin theorem (Feingold and Varga, 1962) is used to determine inclusion regions for the eigenvalues of the overall system in the presence of modeling errors or model simplifications (Solheim, 1981; 1983).

When the block Gerschgorin theorem is used, the inclusion regions for the eigenvalues are usually sharper than those obtained from the usual Gerschgorin circle theorem. The advantages of using the block Gerschgorin theorem in stability studies are that the necessary computations are rather simple and that variations in modeling errors or model simplifications can be studied without much extra computational effort. The eigenvalues of the overall system may, of course, be computed directly. However, the influence of parameter and structure variations on eigenvalue location is then hard to evaluate.

The inclusion regions are in general not very sharp. Therefore, the stability of the system may be better than the method indicates. The use of the block Gerschgorin theorem therefore leads to a conservative design.

Stability close to the trajectory under consideration is guaranteed if all the eigenvalues have negative real parts. In addition to this, the location of the eigenvalues gives an indication of the dynamic properties of the overall system. This is of great interest in robotic manipulators, as oscillatory behaviour is not acceptable in many applications.

The paper is organized as follows: In $\S 2$ the computed torque technique is presented. Then in $\S 3$ the block Gerschgorin theorem is presented. In $\S 4$ the theorem is applied to a general manipulator controlled by the computed torque technique, and an example is given in $\S 5$.

\section{The computed torque technique}

The equations of motion for a general $n$-link manipulator can be found from Newton-Euler's equation (Luh, Walker and Paul, 1980b), and is written

$$
M_{m}(\boldsymbol{q}) \ddot{\boldsymbol{q}}=\boldsymbol{n}_{m}(\boldsymbol{q}, \dot{\boldsymbol{q}})+\boldsymbol{\tau}
$$

where $q$ is the vector of joint coordinates, $M_{m}(q)$ is the inertia matrix for the mechanical part of the manipulator, $n(q, \dot{q})$ is a vector defining friction, Coriolis and centrifugal terms, and $\tau$ is the vector of input generalized forces. Actuator models are given in the form (Paul, 1981)

$$
J_{a} \ddot{q}=-C_{a} \dot{q}-\tau+B_{a} u_{a}
$$

where $\boldsymbol{u}_{a}$ is the vector of actuator control variables and $J_{a}, C_{a}$ and $B_{a}$ are constant diagonal matrices. Combining (1) and (2), and normalizing the input coefficients to unity, we get the state space model

$$
\begin{aligned}
\dot{x}_{1} & =x_{2} \\
M\left(x_{1}\right) \dot{x}_{2} & =n(x)+u_{a}
\end{aligned}
$$

where

$$
\begin{aligned}
x & =\left[x_{1}^{T}, x_{2}^{T}\right], \quad x_{1}=q, \quad x_{2}=\dot{q}, \\
M\left(x_{1}\right) & =M_{m}\left(x_{1}\right)+J_{a}
\end{aligned}
$$


and

$$
n(x)=n_{m}\left(x_{1}, x_{2}\right)+C_{a} x_{2}
$$

By choosing

$$
\boldsymbol{u}_{a}=M\left(\boldsymbol{x}_{1}\right) \boldsymbol{u}-\boldsymbol{n}(\boldsymbol{x})
$$

we get the linear time invariant state space model

$$
\begin{aligned}
& \dot{x}_{1}=x_{2} \\
& \dot{x}_{2}=u
\end{aligned}
$$

where $\boldsymbol{u}$ is the control vector of the linear system (6), (7).

The problem of finding a controller for the system (3) and (4) is now reduced to the much simpler problem of finding a controller to each of the $n$ subsystems

$$
\begin{aligned}
& \dot{x}_{1 i}=x_{2 i} \\
& \dot{x}_{2 i}=u_{i}
\end{aligned}
$$

of (6) and (7). The actuator control vector $u_{a}$ is then given by (5).

In this paper we assume that $u_{i}$ is generated by linear state feedback from $x_{1 i}$ and $x_{2 i}$ as

$$
u_{i}=G_{i i}\left[\Delta x_{1 i}, \Delta x_{2 i}\right]^{T}
$$

where $G_{i i}=\left[g_{i 1}, g_{i 2}\right]$ is the feedback gain matrix for subsystem $i$. $G_{i i}$ can be chosen from classical servomechanism theory, pole-placement techniques or linear quadratic optimal control. The control vector $u$ is then given by

$$
\boldsymbol{u}=G \Delta \boldsymbol{x}
$$

where

$G_{1}=\operatorname{diag}\left(g_{11}, \ldots, g_{n 1}\right)$ and $G_{2}=\operatorname{diag}\left(g_{12}, \ldots, g_{n 2}\right)$, and $\Delta x=\left[\Delta x_{1}^{T}, \Delta x_{2}^{T}\right]^{T}$, where $\Delta x_{1}=x_{1}-x_{10}, \quad \Delta x_{2}=x_{2}-x_{20}, \quad x_{1}=\left[x_{11}, \ldots, x_{1 n}\right]^{T}, \quad x_{2}=\left[x_{21}, \ldots, x_{2 n}\right]^{T}$, $x_{10}=\left[x_{11,0}, \ldots, x_{1 n, 0}\right]^{T}$, and $x_{20}=\left[x_{21,0}, \ldots, x_{2 n, 0}\right]^{T}$.

We now consider the computed torque technique in the presence of modeling errors or model simplifications. The actuator control is now

$$
u_{a}=M_{C}\left(x_{1}\right) u-n_{C}(x)
$$

where $M_{C}$ and $n_{C}$ are the computed values of $M$ and $n$, respectively. The state space model is then found by inserting (12) into (4):

$$
\begin{aligned}
& \dot{x}_{1}=x_{2} \\
& \dot{x}_{2}=M\left(x_{1}\right)^{-1} \Delta n(x)+M\left(x_{1}\right)^{-1} M_{C}\left(x_{1}\right) G \Delta x
\end{aligned}
$$

where $\Delta \boldsymbol{n}=\boldsymbol{n}-\boldsymbol{n}_{\boldsymbol{C}}$.

The system (13), (14) is linearized around a trajectory $x_{1}(t), x_{2}(t)$. The linearized system is

$$
\left[\begin{array}{l}
\Delta \dot{x}_{1} \\
\Delta \dot{x}_{2}
\end{array}\right]=\left[\begin{array}{c:c}
0 & I \\
\hdashline A_{1} & A_{2}
\end{array}\right]\left[\begin{array}{l}
\Delta x_{1} \\
\Delta x_{2}
\end{array}\right]
$$

where

$$
A_{1}=M^{-1}\left(\frac{\partial \Delta n}{\partial x_{1}}+M_{C} G_{1}\right)
$$


and

$$
A_{2}=M^{-1}\left(\frac{\partial \Delta n}{\partial x_{2}}+M_{C} G_{2}\right)
$$

The state vector $\tilde{\boldsymbol{x}}=\left[x_{11}, x_{21}, \ldots, x_{1 n}, x_{2 n}\right]^{T}$ is selected for use in the robustness analysis in Section 4. The linearized state space model (15), (16) is written as

$$
\tilde{\boldsymbol{x}}=\boldsymbol{F} \tilde{\boldsymbol{x}}
$$

where

$$
\begin{aligned}
F & =\left[\begin{array}{ccc}
F_{11} & \ldots & F_{1 n} \\
& \ddots & \\
F_{n 1} & \ldots & F_{n n}
\end{array}\right], \\
F_{i i} & =\left[\begin{array}{cc}
0 & 1 \\
\left(A_{1}\right)_{i i} & \left(A_{2}\right)_{i i}
\end{array}\right]
\end{aligned}
$$

and

$$
F_{i j}=\left[\begin{array}{cc}
0 & 0 \\
\left(A_{1}\right)_{i j} & \left(A_{2}\right)_{i j}
\end{array}\right], \quad i \neq j
$$

We see from (11), (13) and (14) that when $M_{C}=M$ and $\Delta n=0$, we have the nominal system matrix $F_{\text {nom }}$ where

$$
\left(F_{\mathrm{nom}}\right)_{i i}=\left[\begin{array}{cc}
0 & 1 \\
g_{i 1} & g_{i 2}
\end{array}\right]
$$

and

$$
\left(F_{\text {nom }}\right)_{i j}=\left[\begin{array}{ll}
0 & 0 \\
0 & 0
\end{array}\right], \quad i \neq j
$$

\section{The block Gerschgorin theorem}

The block Gerschgorin theorem was first presented by Feingold and Varga (1962). The theorem has been used by Solheim $(1981,1983)$ in the design of decentralızea control of interconnected dynamic systems and in robustness analysis.

We let $\|\boldsymbol{x}\|$ denote the Euclidean norm

$$
\|\boldsymbol{x}\|=\left[\sum_{i}\left|x_{i}\right|^{2}\right]^{1 / 2}
$$

and $\|A\|$ the matrix norm

$$
\|A\|=\left[\max _{i} \lambda_{i}\left(A^{T} A\right)\right]^{1 / 2}
$$

where $\lambda_{i}\left(A^{T} A\right)$ is an eigenvalue of $A^{T} A$. For a normal matrix $N$, we have $[\lambda(N)]^{2}=\lambda\left(N^{T} N\right)$, and therefore

$$
\|N\|=\max _{i}\left|\lambda_{i}(N)\right|
$$


In the following we need the relation

$$
\left(\left\|N^{-1}\right\|\right)^{-1}=\min _{i}\left|\lambda_{i}(N)\right|
$$

By continuity, we define $\left(\left\|N^{-1}\right\|\right)^{-1}$ to be zero when $N$ is singular. The relation (28) holds as $\lambda\left(A^{-1}\right)=[\lambda(A)]^{-1}$ for any non-singular matrix $A$.

We consider the $n \times n$ matrix $A$ which is partitioned in the following manner:

$$
A=\left[\begin{array}{ccc}
A_{11} & \ldots & A_{1 N} \\
& \ddots & \\
A_{N 1} & \ldots & A_{N N}
\end{array}\right]
$$

where the diagonal sub-matrices $A_{i i}, i=1, \ldots, N$ are square of order $n_{i}$.

The block Gerschgorin theorem then states that for the partitioned matrix $A$ of (29), each eigenvalue of $A$ satisfies

$$
\left(\left\|\left(A_{j j}-\lambda I_{j}\right)^{-1}\right\|\right)^{-1} \leqslant \sum_{\substack{k=1 \\ k \neq j}}^{N}\left\|A_{j k}\right\|
$$

for at least one $j, 1 \leqslant j \leqslant N$.

Here $I_{j}$ is the $n_{j} \times n_{j}$ identity matrix.

When the diagonal sub-matrices are normal, we get from (28)

$$
\left(\left\|\left(A_{j j}-\lambda I_{j}\right)^{-1}\right\|\right)^{-1}=\min _{i}\left|\sigma_{i}-\lambda\right|
$$

where $\sigma_{i}$ is an eigenvalue of $A_{j j}$. The inclusion regions for the eigenvalues of $A$ are then given by

$$
\min _{i}\left|\sigma_{i}-\lambda\right| \leqslant \sum_{\substack{k=1 \\ k \neq 1}}^{N}\left\|A_{j k}\right\|
$$

We see that when the diagonal sub-matrices are normal, the eigenvalues of $A$ will for at least one $j, 1 \leqslant j \leqslant N$, be included in the union of circles that have their centre in the eigenvalues of $A_{j j}$ and a radius that is equal to the right side of (31).

\section{Determination of inclusion regions for the eigenvalues in the} presence of model errors

In this section the stability of the linear perturbation model (19) is analysed in terms of its eigenvalues.

We consider the system matrix $F_{\text {nom }}$ with perturbations $\pm \Delta F . F_{\text {nom }}$ is given by (23) and (24). We establish an augmented system matrix (Solheim, 1983)

$$
F_{\text {aug }}=\left[\begin{array}{c:c}
F_{\text {nom }}+\Delta F & 0 \\
\hdashline 0 & F_{\text {nom }}-\Delta F
\end{array}\right]
$$

We use the similarity transformation

$$
S^{-1} F_{\text {aug }} S=\left[\begin{array}{c:c}
F_{\text {nom }} & \Delta F \\
\hdashline \Delta F & F_{\text {nom }}
\end{array}\right]=\bar{F}
$$


where

$$
S=\left[\begin{array}{c:c}
-I & -I \\
\hdashline-I & I
\end{array}\right]
$$

The eigenvalues of $\bar{F}$ consist of the eigenvalues of $F_{\text {nom }}+\Delta F$ and the eigenvalues of $F_{\text {nom }}-\Delta F$. In order to make $\bar{F}$ suitable for the use of the block Gerschgorin theorem, we make the diagonal submatrices normal by the similarity transformation

$$
\left[\begin{array}{c:c}
T^{-1} & 0 \\
\hdashline 0 & T^{-1}
\end{array}\right]\left[\begin{array}{c:c}
F_{\text {nom }} & \Delta F \\
\hdashline \Delta F & F_{\text {nom }}
\end{array}\right]\left[\begin{array}{c:c}
T & 0 \\
\hdashline 0 & T
\end{array}\right]=\tilde{F}
$$

where $T=\operatorname{diag}\left(1, g_{11}^{1 / 2}, 1, g_{21}^{1 / 2}, \ldots, 1, g_{n 1}^{1 / 2}\right)$. This gives

$$
\tilde{F}=\left[\begin{array}{c:c}
T^{-1} F_{\text {nom }} T & T^{-1} \Delta F T \\
\hdashline T^{-1} \Delta F T & T^{-1} F_{\text {nom }} T
\end{array}\right]
$$

where

$$
\begin{aligned}
\left(T^{-1} F_{\text {nom }} T\right)_{i i} & =\left[\begin{array}{cc}
0 & g_{i 1}^{1 / 2} \\
g_{i 1}^{1 / 2} & g_{i 2}
\end{array}\right] \\
\left(T^{-1} F_{\text {nom }} T\right)_{i j} & =\left[\begin{array}{ll}
0 & 0 \\
0 & 0
\end{array}\right], \quad i \neq j \\
\left(T^{-1} \Delta F T\right)_{i i} & =\left[\begin{array}{cc}
0 & 0 \\
g_{i 1}^{-1 / 2}\left[\left(A_{1}\right)_{i i}-g_{i 1}\right] & \left(A_{2}\right)_{i i}-g_{i 2}
\end{array}\right]
\end{aligned}
$$

and

$$
\left(T^{-1} \Delta F T\right)_{i j}=\left[\begin{array}{cc}
0 & 0 \\
g_{i 1}^{-1 / 2}\left(A_{1}\right)_{i j} & \left(g_{j 1} / g_{i 1}\right)^{-1 / 2}\left(A_{2}\right)_{i j}
\end{array}\right], \quad i \neq j
$$

where $A_{1}$ and $A_{2}$ are defined in (19)-(22).

The inclusion regions for the eigenvalues of $F_{\text {aug }}$ are then found from (31) and are given by

$$
\min _{j}\left|\sigma_{j}-\lambda\right| \leqslant r_{j}
$$

where $\sigma_{j}$ is an eigenvalue of $\left(F_{\text {nom }}\right)_{i i}$ and

$$
\begin{aligned}
r_{i}= & \sum_{\substack{j=1 \\
j \neq i}}^{n}\left\{\left[g_{i 1}^{-1}\left(\left(A_{1}\right)_{i j}\right)^{2}+\left(\frac{g_{j 1}}{g_{i 1}}\right)^{-1}\left(\left(A_{2}\right)_{i j}\right)^{2}\right]^{1 / 2}\right\} \\
& +\left[g_{i 1}^{-1}\left(\left(A_{1}\right)_{i i}-g_{i 1}\right)^{2}+\left(\left(A_{2}\right)_{i i}-g_{i 2}\right)^{2}\right]^{1 / 2}
\end{aligned}
$$

\section{Application to the positioning part of manipulator with rotary joints}

We consider the manipulator in Fig. 1 which is the positioning part of an industrial manipulator. The state space model of motion is given by (Saridis, 1983)

$$
\begin{aligned}
\dot{x}_{1} & =x_{2} \\
M\left(x_{1}\right) \dot{x}_{2} & =n(x)+\tau
\end{aligned}
$$

where $\boldsymbol{x}_{1}=\left[q_{1}, q_{2}, q_{3}\right]^{T}, \boldsymbol{x}_{2}=\left[\dot{q}_{1}, \dot{q}_{2}, \dot{q}_{3}\right]^{T}, \tau_{1}=\left[\tau_{1}, \tau_{2}, \tau_{3}\right]^{T}, \tau_{1}, \tau_{2}$ and $\tau_{3}$ are the input joint torques, 


$$
M\left(x_{1}\right)=\left[\begin{array}{ccc}
J_{1} & 0 & 0 \\
0 & J_{22}+2 J_{23} C_{3}+J_{33} & J_{23} C_{3}+J_{33} \\
0 & J_{23} C_{3}+J_{33} & J_{33}
\end{array}\right]
$$

and

$$
J_{1}=J_{11}+J_{22} C_{2}^{2}+J_{33} C_{2+3}^{2}+J_{23} C_{2} C_{2+3}
$$

$\boldsymbol{n}=\left[n_{1}, n_{2}, n_{3}\right]^{T}$ where

$$
\begin{aligned}
n_{1}= & 2\left[J_{23} S_{2} C_{2+3}+J_{22} S_{2} C_{2}\right] \dot{q}_{1} \dot{q}_{2} \\
& +2\left[J_{23} S_{2+3} C_{2}+J_{33} S_{2+3} C_{2+3}\right] \dot{q}_{1}\left(\dot{q}_{2}+\dot{q}_{3}\right) \\
n_{2}= & -\left[J_{23}\left(C_{2+3} S_{2}+C_{2} S_{2+3}\right)+J_{22} C_{2} S_{2}+J_{33} C_{2+3} S_{2+3}\right] \dot{q}_{1}^{2} \\
& +J_{23} S_{3} \dot{q}_{3}^{2}+M_{2} C_{2}+2 M_{3} C_{2+3} \\
n_{3}= & -\left[J_{23} C_{2} S_{2+3}+J_{33} C_{2+3} S_{2+3}\right] \dot{q}_{1}^{2}-J_{23} S_{3} \dot{q}_{2}^{2}+M_{3} C_{2+3}
\end{aligned}
$$

Here $C_{2}=\cos q_{2}, \quad C_{2+3}=\cos \left(q_{2}+q_{3}\right), \quad C_{3}=\cos q_{3}, \quad S_{2}=\sin q_{2}, \quad S_{2+3}=$ $\sin \left(q_{2}+q_{3}\right)$ and $S_{3}=\sin q_{3}$.

For the robot in our study, the moments of inertia are

$$
\begin{aligned}
& J_{11}=2.0 \\
& J_{22}=18.1 \\
& J_{23}=8.0 \\
& J_{33}=6.67
\end{aligned}
$$

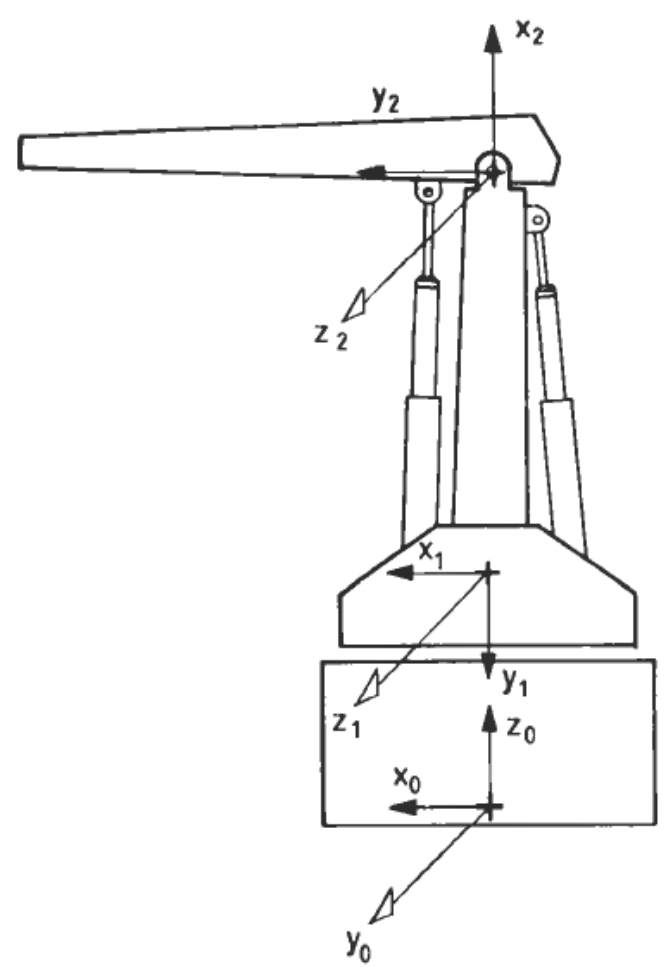

Figure 1. Manipulator. 
and the gravitational coefficients are

$$
\begin{aligned}
& M_{2}=12 \cdot 5 \\
& M_{3}=10 \cdot 0
\end{aligned}
$$

Here we consider the joint torques to be our control variables. This will be the case when direct current motors with current feedback or hydraulic motors with pressure feedback are used. In the case of voltage controlled direct current motors, we see from (3) and (4) that although the parameters of the model are changed, the structure is the same.

We use the computed torque technique, and choose

$$
\tau=-n_{C}(x)+M_{C}\left(x_{1}\right) G \Delta x
$$

The feedback gain matrix $G$ is chosen as

$$
G=\left[\begin{array}{l:l}
-100 I_{3} & -20 I_{3}
\end{array}\right]
$$

The eigenvalues of the diagonal submatrices of $F_{\text {nom }}$ will then be $\sigma_{1}=\sigma_{2}=-10$.

We first consider the case where the compensation for the nonlinear terms is inaccurate, that is $\Delta n \neq 0$, while the inertia matrix $M_{C}=M$.

The inclusion regions were computed for $\ddot{q}_{1}=\ddot{q}_{2}=\ddot{q}_{3}=0$ and $\left|\dot{q}_{i}\right| \leqslant 1 \mathrm{rad} / \mathrm{s}$, $i=1, \ldots, 3$ from eqns (17), (18), (42) and (44)-(49). The nominal eigenvalues were located at $\sigma_{1}=\sigma_{2}=-10$, which means that when the radius of the largest inclusion region is less than 10 , the eigenvalues will be in the left half plane. When link three is horizontal, that is $q_{2}+q_{3}=0^{\circ}$, and $30^{\circ}<q_{2}<150^{\circ}$, the largest radius is $r=3 \cdot 0$ (Fig. 2).

In Fig. 3 the inclusion region is shown when $q_{2}=60^{\circ}$ and $q_{2}+q_{3}=0^{\circ}$.

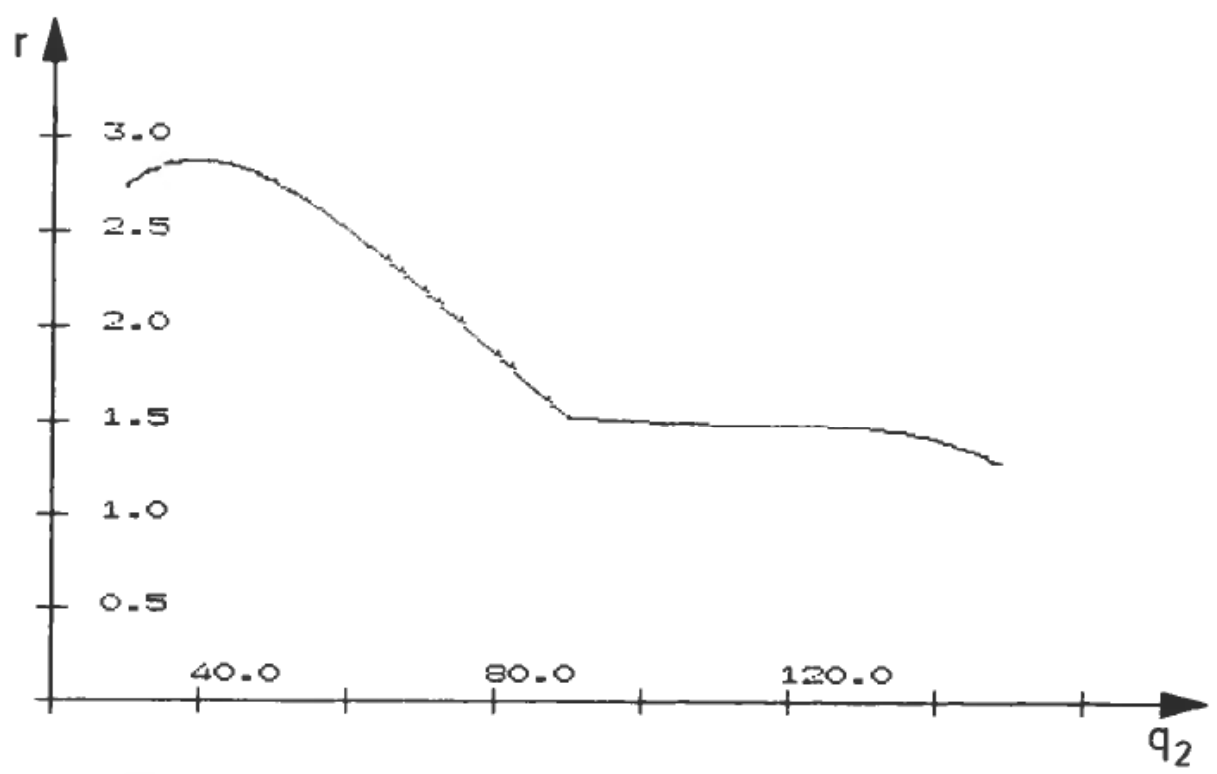

Figure 2. Radius of inclusion region when $\left|\dot{q}_{i}\right| \leqslant 1 \mathrm{rad} / \mathrm{s}, i=1,2,3$, and link 3 is horizontal $\left(q_{2}+q_{3}=0^{\circ}\right)$. 


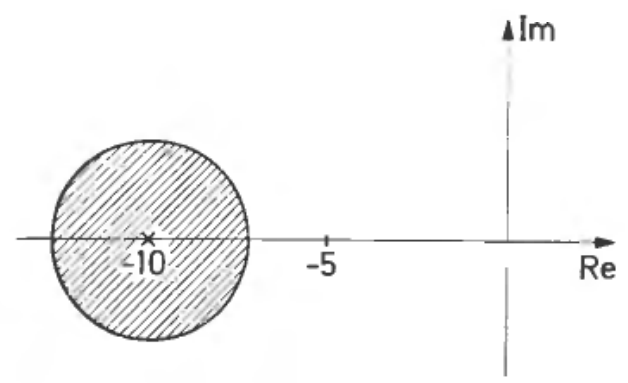

Figure 3. Inclusion region when $\left|\dot{q}_{i}\right| \leqslant 1 \mathrm{rad} / \mathrm{s}, i=1,2,3, q_{2}=60^{\circ}$ and $q_{3}=-60^{\circ}$.

When link two is vertical, that is $q_{2}=90^{\circ}$, and $-60^{\circ}<q_{2}+q_{3}<20^{\circ}$, the largest radius is still $r=3.0$. When $q_{1}=90^{\circ}$ and $q_{2}+q_{3}$ increases to $60^{\circ}, r$ increases to 9.0 (Fig. 4).

We see that even the large error $\Delta \boldsymbol{n}=\boldsymbol{n}$ has little influence on the eigenvalue location except when $q_{2}=90^{\circ}$ and $q_{2}+q_{3}$ approaches $60^{\circ}$, but even here the eigenvalues are in the left half plane.

We now consider an inaccurate $M_{C}$ when $\boldsymbol{n}=\mathbf{0}$ and examine the eigenvalue location when $q_{1}=60^{\circ}$ and $q_{3}=-60^{\circ}$. A diagonal $M_{C}$ is investigated first. We let

$$
M_{C}=\left[\begin{array}{ccc}
\alpha 21 \cdot 7 & 0 & 0 \\
0 & 32 \cdot 77 & \beta 10 \cdot 7 \\
0 & \beta 10 \cdot 7 & 6.7
\end{array}\right]
$$

which is equal to $M$ when $\alpha=1$ and $\beta=1$. We then get

$$
A_{1}-G_{1} \cong\left[\begin{array}{ccc}
100(\alpha-1) & 0 & 0 \\
0 & 210-110 \beta & 67(\beta-1) \\
0 & 320(\beta-1) & 200-100 \beta
\end{array}\right]
$$

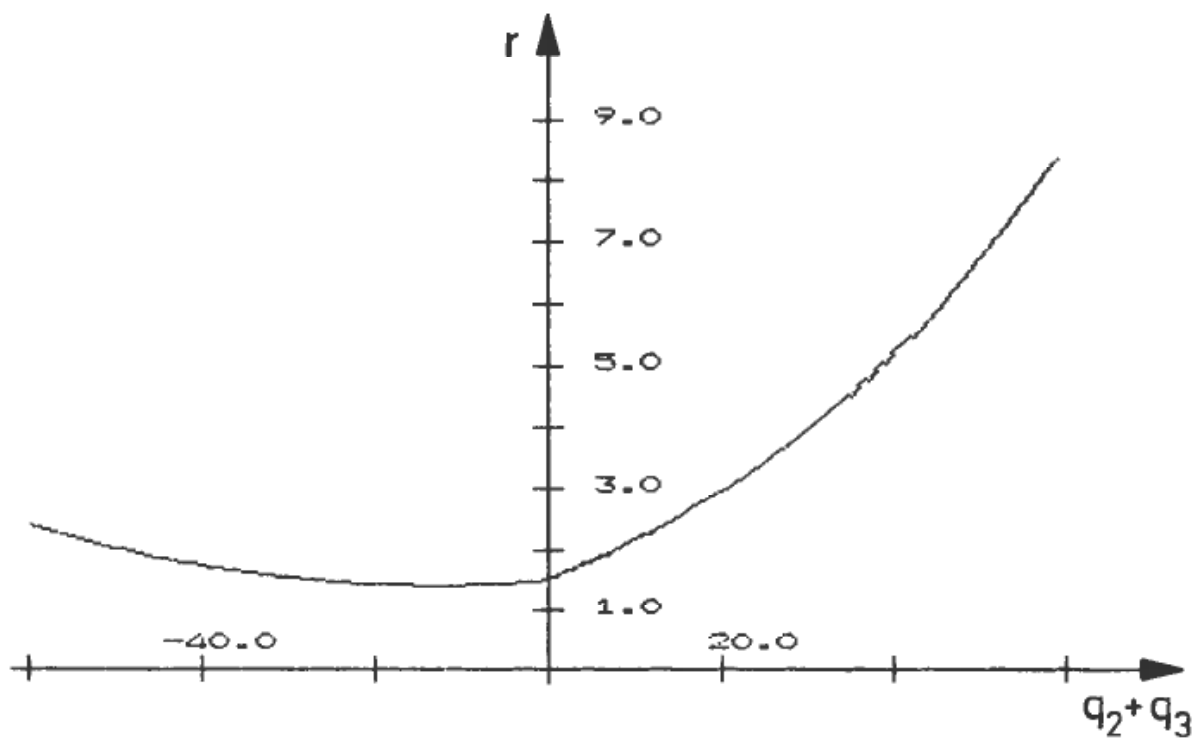

Figure 4. Radius of inclusion region when $=\dot{q}_{i} \mid \leqslant 1 \mathrm{rad} / \mathrm{s}, i=1,2,3$, and link 2 is vertical $\left(q_{2}=90^{\circ}\right)$. 


$$
A_{2}-G_{2}=\left[\begin{array}{ccc}
20(\alpha-1) & 0 & 0 \\
0 & 22(1-\beta) & 13 \cdot 4(\beta-1) \\
0 & 64(\beta-1) & 1-\beta
\end{array}\right]
$$

When $\alpha=1$, the condition that guarantees stability, $r_{i}<10, i=1,2,3$ is satisfied for $0.85<\beta<1.15$. For $\beta=1$, we have $r_{i}<10, i=1,2,3$ when $0.5<\alpha<2$. This means that even small changes in $M_{C}$ may result in instability, while relatively large $\Delta \boldsymbol{n}$ is tolerated.

\section{Conclusion}

It has been demonstrated that the block Gerschgorin theorem is suited for robustness analysis of the computed torque technique. The theorem las been applied to the positioning part of an industrial manipulator. The results indicate that errors as large as the correct compensation may be tolerated in the non-linear feedback compensation of system non-linearities. However, the eigenvalue location seems to be very sensitive to errors in the computed inertia matrix.

This result is reasonable as the $M_{C}$ matrix enters multiplicatively in the $M^{-1} M_{C} G$ term in (14), while $\Delta n$ enters only in the $M^{-1} \partial \Delta n / \partial x$ term. This means that $M_{C}$ enters multiplicatively in the feedback gain, while $\partial \Delta n / \partial x$ only enters additively as a small term in the feedback gain.

\section{ACKNOWLEDGMENT}

This work has been sponsored by the Royal Norwegian Council for Scientific and Industrial Research through the Robotics research program ED331.12510.

\section{REFERENCES}

BEJCZY, A. K. (1974). Robot arm dynamics and control. JPL Technical Memo, 33-369.

FeINGOLD, D. G. and VARGA, R. S. (1962). Block diagonally dominant matrices and generalization of the Gerschgorin circle theorem. Pacific J. Math., 12, 1241-1250.

LuH, J. Y. S., WalKer, M. W., and Paul, R. P. C. (1980a). Resolved acceleration control of mechanical manipulators. I.E.E.E. Trans. Automatic Control, 25, 468-474.

LuH, J. Y. S., Walker, M. W., and Paul, R. P. C. (1980b). On-line computational scheme for mechanical manipulators. J. Dynamic Systems, Measurement and Control, 102, 69-76.

LUo, G. L. and SARIDIS, G. N. (1985). Optimal/PID formulation for control of robotic manipulators. Proc. 1985 I.E.E.E. Int. Conference on Robotics and Automation, St. Louis, Missouri, March 25-28, 1985, 621-626.

PAUL, R. P. (1981). Robot Manipulators: Mathematics, Programming and Control (The MIT press; Cambridge, Massachusetts).

SARIDIS, G. N. (1983). Intelligent robotic control. I.E.E.E. Trans. Automatic Control, 28, 547557.

Solmeim, O. A. (1981). On the use of a block analogue of the Gerschgorin circle theorem in the design of decentralized control of a class of large scale systems. 2nd IFAC Symposium on large scale systems, Toulouse, June 24-26, 1980. Modeling, Identification and Control, 2, 107-118.

Solneim, O. A. (1983). Robustness analysis of a class of optimal control systems. Modeling, Identification and Control, 4, 223-236.

SpONG, M. W. and VidYASAGAR, M. (1985). Robust linear compensator design for non-linear robotic control. Proc. 1985 I.E.E.E. Int. Conference on Robotics and Automation, St. Louis, Missouri, March 25-28, 1985, 621-626.

TARN, T. J., BeJCZY, A. K., IsIDORI, A., and ChEN, Y. (1984). Non-linear feedback in robot arm control. Proc. 23rd I.E.E.E. Conference on Decision and Control, Las Vegas, Nevada, Dec. 12-14, 1984. 